\title{
Numerical Analytic Solution of SIR Model of Dengue Fever Disease in South Sulawesi using Homotopy Perturbation Method and Variational Iteration Method
}

\author{
Yulita Molliq Rangkuti ${ }^{1}$, Syafruddin $\operatorname{Side}^{2} \&$ Mohd Salmi Md Noorani ${ }^{3}$ \\ ${ }^{1}$ Department of Mathematics, Faculty of Mathematics and Natural Sciences, \\ Universitas Negeri Medan, Jalan Willem Iskandar, \\ Pasar V Medan Estate, Medan 20221, Indonesia \\ ${ }^{2}$ Department of Mathematics, Faculty of Mathematics and Natural Sciences, \\ Universitas Negeri Makasar, Jalan A.P. Pettarani, Makasar 90245, Indonesia \\ ${ }^{3}$ School of Mathematical Sciences, Faculty of Science and Technology, \\ Universiti Kebangsaan Malaysia, 43600 Bangi, Selangor, Malaysia \\ Email: yulitamolliq@yahoo.com
}

\begin{abstract}
In this research, the susceptible-infected-recovered (SIR) model of dengue fever is considered. We have implemented two analytical techniques, namely the variational iteration method (VIM) and the homotopy perturbation method (HPM) for solving the SIR model. The Lagrange multiplier was investigated for the VIM and He's polynomial approach for the HPM was used. In these schemes, the solution takes the form of a convergent series with easily computable components. The results show that the VIM solution is more accurate than the HPM solution for short time intervals, whereas the HPM is more accurate than the VIM for long time intervals when compared with the fourth-order Runge-Kutta method (RK4).We found that the HPM and the RK4 were in excellent conformance.
\end{abstract}

Keywords: He's polynomial; homotopy perturbation method; Lagrange multiplier; SIR model; variational iteration method.

\section{Introduction}

Dengue fever is regarded as a serious infectious disease, threatening about 2.5 billion people all over the world, especially in tropical countries. Dengue fever has become a major epidemic disease in Southeast Asia. This epidemic can be described to climate change and is made worse by the population's lack of knowledge about and awareness of dengue fever, so that dengue fever may become endemic [1]. Thus, building a dengue fevermodel is important.

Mathematical models for dengue fever have investigated compartment dynamics using susceptible, infected, and removed (SIR) models [2-7]; these models only scrutinized the formulation of the model. Side and Noorani [1] have modified the models in [8] and [9] and applied real data as reported by the

Received August $20^{\text {th }}, 2013,1^{\text {st }}$ Revision March $5^{\text {th }} 2014,2^{\text {nd }}$ Revision April $9^{\text {th }}, 2014$ Accepted for publication April $14^{\text {th }}, 2014$.

Copyright $\odot 2014$ Published by ITB Journal Publisher, ISSN: 2337-5760, DOI: 10.5614/j.math.fund.sci.2014.46.1.8 
Ministry of Health in South Sulawesi, Indonesia (KKRI) [8]. Side and Noorani [1] also matched empirical data with a simulation model. Hence, the SIR model presented in [1] was intended to be a trusted reference and control tool when dealing with dengue fever in South Sulawesi. To find the number of spreading populations in this model [1] using a semi-numerical method is interesting to investigate. The right method must be chosen to solve this model.

In this study, the homotopy perturbation method (HPM) and the variational iteration method (VIM) which were proposed by $\mathrm{He}$ in [10,11] and [9] respectively, were used for solving the SIR model of dengue fever. The Lagrange multiplier was investigated for the VIM and He's polynomial approach for the HPM was used. Over the years, the VIM and HPM have grown into a widely appreciated method. Several researchers have shown further applications. Yulita and colleagues [12-14] obtained the approximate solution of fractional heat and wave-like equations, fractional Zakharov-Kuznetsov equations and fractional Rosenao-Hayman equation using the VIM. Yulita, et al. [15] modified the VIM to find the approximate solution of a fractional biochemical reaction model. Rafei, et al. [16] applied the VIM for solving the epidemic model and the prey and predator problem. Khan, et al. [17] applied the HPM to the Vector Host Epidemic Model with Non-Linear Incidences and Ghotbi, et al. [18] applied the HPM and the VIM to the SIR epidemic model. Recently, Islam, et al. [19] obtained the analytical solution of an SEIV epidemic model using the HPM.

The procedure of the two methods for the SIR model will be discussed later. In this paper, a comparison between the fourth-order Runge-Kutta method (RK4) and collected data in [1] will be made. From the Figure 1, the authors decided to choose the RK4 solution as benchmark for the SIR model of dengue fever. Furthermore, the VIM and HPM solutions will also be matched with the RK4 solution to show the accuracy of the methods.

\section{Susceptible Infected Recovery (SIR) of Dengue Fever in South Sulawesi}

Side and Noorani [1] defined a SIR model of dengue fever in the following equations:

$$
\begin{aligned}
& \frac{d x}{d t}=\mu_{h}(1-x(t))-\alpha x(t) z(t), \\
& \frac{d y}{d t}=\alpha x(t) z(t)-\beta y(t), \\
& \frac{d z}{d t}=\gamma(1-z(t)) y(t)-\delta_{1} z(t),
\end{aligned}
$$


where $\quad x=\frac{S_{h}}{N_{h}}, y=\frac{I_{h}}{N_{h}}, z=\frac{I_{v}}{N_{v}}=\frac{I_{v}}{A / \mu_{v}}, \alpha=\frac{b \beta_{h} A}{\mu_{v} N_{h}}, \beta=\gamma_{h}+\mu_{h} \quad$ and $\quad \gamma=$ $b \beta_{v}, \delta=\mu_{v}$.

$N_{h}$ is the human population, $S_{h}$ is people who may potentially get infected with the dengue virus, $I_{h}$ is people who are infected with dengue, $R_{h}$ is people who have recovered. The vector population of mosquitoes $\left(N_{v}\right)$ is divided into two groups: mosquitoes that may potentially become infected with the dengue virus (susceptible; $S_{v}$ ) and mosquitoes that are infected with the dengue virus $\left(I_{v}\right)$. $b \beta_{h}$ is the sufficient rate of correlation between the vector population and the human population.

\section{Homotopy Perturbation Method}

To implement HPM, first, we write the general system of differential equations in the operator form:

$$
\begin{aligned}
& \frac{d u_{1}}{d t}+g_{1}\left(t, u_{1}, u_{2}, \cdots, u_{m}\right)=f_{1}(t), \\
& \frac{d u_{2}}{d t}+g_{2}\left(t, u_{1}, u_{2}, \cdots, u_{m}\right)=f_{2}(t), \\
& \frac{d u_{m}}{d t}+g_{m}\left(t, u_{1}, u_{2}, \cdots, u_{m}\right)=f_{m},
\end{aligned}
$$

subject to the initial conditions

$$
u_{1}\left(t_{0}\right)=c_{1}, \quad u_{2}\left(t_{0}\right)=c_{2}, \quad \cdots u_{m}\left(t_{0}\right)=c_{m} .
$$

Then we write system (4)-(6) in the following operator form:

$$
\begin{aligned}
& L\left(u_{1}\right)+N_{1}\left(u_{1}, u_{2}, \cdots, u_{m}\right)-f_{1}(t)=0, \\
& L\left(u_{2}\right)+N_{2}\left(u_{1}, u_{2}, \cdots, u_{m}\right)-f_{2}(t)=0, \\
& L\left(u_{2}\right)+N_{2}\left(u_{1}, u_{2}, \cdots, u_{m}\right)-f_{2}(t)=0,
\end{aligned}
$$

subject to the initial conditions (7), where $L=d / d t$ is a linear operator and $N_{1}, N_{2}, \ldots, N_{\mathrm{n}}$ are nonlinear operators. Next, we will present the solution approaches of (8)-(10) based on the standard HPM.

According to HPM, we construct a homotopy for (8)-(10) that satisfies the following relations:

$$
\begin{aligned}
& L\left(u_{1}\right)-L\left(v_{1}\right)+p L\left(v_{1}\right)+p\left[N_{1}\left(u_{1}, u_{2}, \cdots, u_{m}\right)-f_{1}(t)\right]=0, \\
& L\left(u_{2}\right)-L\left(v_{2}\right)+p L\left(v_{2}\right)+p\left[N_{2}\left(u_{1}, u_{2}, \cdots, u_{m}\right)-f_{2}(t)\right]=0, \\
& L\left(u_{m}\right)-L\left(v_{m}\right)+p L\left(v_{m}\right)+p\left[N_{m}\left(u_{1}, u_{2}, \cdots, u_{m}\right)-f_{m}(t)\right]=0,
\end{aligned}
$$


where $p \in[0,1]$ is an embedding parameter and $v_{1}, v_{2}, \ldots, v_{m}$ are initial approximations that satisfy the given conditions. It is obvious that when perturbation parameter $p=0$, Eqs. (11)-(13) become a linear system of equations and when $p=1$ we get the original nonlinear system of equations. Let us take the initial approximations as follows:

$$
\begin{aligned}
& u_{1,0}(t)=v_{1}(t)=u_{1}\left(t_{0}\right)=c_{1}, \\
& u_{2,0}(t)=v_{2}(t)=u_{2}\left(t_{0}\right)=c_{2}, \\
& u_{m, 0}(t)=v_{m}(t)=u_{m}\left(t_{0}\right)=c_{m} .
\end{aligned}
$$

and

$$
\begin{aligned}
& u_{1}(t)=u_{1,0}(t)+p u_{1,1}(t)+p^{2} u_{1,2}(t)+\cdots, \\
& u_{2}(t)=u_{2,0}(t)+p u_{2,1}(t)+p^{2} u_{2,2}(t)+\cdots, \\
& u_{m}(t)=u_{m, 0}(t)+p u_{m, 1}(t)+p^{2} u_{m, 2}(t)+\cdots,
\end{aligned}
$$

where $u_{i, j}(i=1,2, \ldots, m ; j=1,2, \ldots)$ are functions yet to be determined. Substituting (14)-(19) into (11)-(13) and arranging the coefficients of the same powers of $p$, we get

$$
\begin{aligned}
& L\left(u_{1,1}\right)+L\left(v_{1}\right)+N_{1}\left(u_{1,0}, u_{2,0}, \cdots, u_{m, 0}\right)-f_{1}=0, u_{1,1}\left(t_{0}\right)=0, \\
& L\left(u_{2,1}\right)+L\left(v_{2}\right)+N_{2}\left(u_{1,0}, u_{2,0}, \cdots, u_{m, 0}\right)-f_{2}=0, u_{2,1}\left(t_{0}\right)=0, \\
& L\left(u_{m, 1}\right)+L\left(v_{m}\right)+N_{m}\left(u_{1,0}, u_{2,0}, \cdots, u_{m, 0}\right)-f_{m}=0, u_{m, 1}\left(t_{0}\right)=0,
\end{aligned}
$$

and

$$
\begin{aligned}
& L\left(u_{1,2}\right)+N_{1}\left(u_{1,0}, u_{2,0}, \cdots, u_{m, 0}\right)-f_{1}=0, u_{1,2}\left(t_{0}\right)=0, \\
& L\left(u_{2,2}\right)+N_{2}\left(u_{1,0}, u_{2,0}, \cdots, u_{m, 0}\right)-f_{2}=0, u_{2,2}\left(t_{0}\right)=0, \\
& L\left(u_{m, 2}\right)+N_{m}\left(u_{1,0}, u_{2,0}, \cdots, u_{m, 0}\right)-f_{m}=0, u_{m, 2}\left(t_{0}\right)=0,
\end{aligned}
$$

etc. We solve the above systems of equations for the unknowns $u_{i, j}(i=$ $1,2, \ldots, m ; j=1,2, \ldots)$ by applying the inverse operator

$$
L^{-1}(\cdot)=\int_{0}^{t}(\cdot) d t .
$$

Therefore, according to HPM the $n$-term approximations to the solutions of (8)(10) can be expressed as

$$
\begin{aligned}
& \phi_{1, n}(t)=u_{1}(t)=\lim _{p \rightarrow 1} u_{1}(t)=\sum_{k=0}^{n-1} u_{1, k}(t), \\
& \phi_{2, n}(t)=u_{2}(t)=\lim _{p \rightarrow 1} u_{2}(t)=\sum_{k=0}^{n-1} u_{2, k}(t), \\
& \phi_{m, n}(t)=u_{m}(t)=\lim _{p \rightarrow 1} u_{m}(t)=\sum_{k=0}^{n-1} u_{m, k}(t),
\end{aligned}
$$




\section{$4 \quad$ Variational Iteration Method (VIM)}

To introduce the basic concepts of VIM, we consider the following nonlinear differential equation:

$$
L u_{i}(t)+N u_{i}(t)=g_{i}(t), \quad i=1,2, \cdots, n
$$

where $L$ is a linear operator, $N$ is a nonlinear operator, and $g_{i}(t)$ is an inhomogeneous term. According to VIM, one can construct a correction functional as follows:

$$
u_{i, n+1}=u_{i, n}+\int_{0}^{t} \lambda_{i}(s)\left[L u_{i, n}(s)+N \widetilde{u_{l, n}}(s)-g_{i}(t)\right] d s,
$$

where $\lambda_{i} i=1,2, \cdots, n$ are the Lagrange multipliers [20], which can be identified optimally via the variational theory, and $\widetilde{u_{l, n}}(s)$ are considered as restricted variations, i.e. $\widetilde{\delta u_{l, n}}(s)=0$. Once we have determined the Lagrange multiplier, we use VIM to perform the iteration using the initial approximation, which we choose by a linearized solution of the equation that satisfies the initial conditions. Therefore, we can successively approximate or even reach the exact solution by using

$$
u(t)=\lim _{n \rightarrow \infty} u_{i, n}(t)
$$

\section{Implementation of HPM}

First, write the SIR model of dengue feverin the following form:

$$
\begin{aligned}
& \frac{d x}{d t}=\mu_{h}(1-x(t))-\alpha x(t) z(t) \\
& \frac{d y}{d t}=\alpha x(t) z(t)-\beta y(t) \\
& \frac{d z}{d t}=\gamma(1-z(t)) y(t)-\delta_{1} z(t),
\end{aligned}
$$

subject to the initial conditions

$$
x\left(t_{0}\right)=c_{1}, \quad y\left(t_{0}\right)=c_{2}, z\left(t_{0}\right)=c_{3},
$$

Then we write system (30)-(32) in the operator form:

According to HPM, we construct a homotopy for (33)-(35) that satisfies the following relations:

$$
\begin{aligned}
& v_{1}^{\prime}-x_{0}^{\prime}+p\left(x_{0}^{\prime}-\mu_{h}\left(1-v_{1}\right)+\alpha v_{1} v_{3}\right)=0, \\
& v_{2}^{\prime}-y_{0}^{\prime}+p\left(y_{0}^{\prime}{ }_{0}-\alpha v_{1} v_{3}+\beta v_{2}\right)=0, \\
& v_{3}^{\prime}-z_{0}^{\prime}+p\left(z^{\prime}{ }_{0}-\gamma\left(1-v_{3}\right) v_{2}+\delta_{1} v_{3}\right)=0 .
\end{aligned}
$$


Let us choose the initial approximations as

$$
\begin{aligned}
& v_{1,0}(t)=x_{0}(t)=v_{1}(0)=c_{1}, \\
& v_{2,0}(t)=y_{0}(t)=v_{2}(0)=c_{2}, \\
& v_{3,0}(t)=z_{0}(t)=v_{3}(0)=c_{3},
\end{aligned}
$$

and

$$
\begin{aligned}
& v_{1}(t)=v_{1,0}(t)+p v_{1,1}(t)+p^{2} v_{1,2}(t)+p^{3} v_{1,3}(t)+\cdots, \\
& v_{2}(t)=v_{2,0}(t)+p v_{2,1}(t)+p^{2} v_{2,2}(t)+p^{3} v_{2,3}(t)+\cdots, \\
& v_{3}(t)=v_{3,0}(t)+p v_{3,1}(t)+p^{2} v_{3,2}(t)+p^{3} v_{3,3}(t)+\cdots,
\end{aligned}
$$

where $v_{i, j} i(i=1,2 ; j=1,2,3, \ldots)$ are functions yet to be determined. Substituting into and collecting terms of the same powers of $p$, we have

$$
\begin{gathered}
v_{1,1}^{\prime}+\alpha v_{1,0} v_{3,0}+\mu_{h} v_{1,0}-\mu_{h}=0, v_{1,1}(0)=0 \\
v_{2,1}^{\prime}-\alpha v_{1,0} v_{3,0}+\beta v_{2,0}=0, \quad v_{2,1}(0)=0 \\
v_{3,1}^{\prime}-\gamma v_{2,0}+\delta_{1} v_{3,0}+\gamma v_{2,0} v_{3,0}=0 v_{3,1}(0)=0 \\
v_{1,2}^{\prime}+\mu_{h} v_{1,1}+\alpha v_{1,1} v_{3,0}+\alpha v_{1,0} v_{3,1}=0 v_{1,2}(0)=0 \\
v_{2,2}^{\prime}+\beta v_{2,1}-\alpha v_{1,1} v_{3,0}-\alpha v_{1,0} v_{3,1}=0, \quad v_{2,2}(0)=0 \\
v_{3,2}^{\prime}-\gamma v_{2,1}+\gamma v_{2,1} v_{3,0}+\delta_{1} v_{3,1}+\gamma v_{2,0} v_{3,1}=0, v_{3,2}(0)=0 \\
v_{1,3}^{\prime}+\alpha v_{1,0} v_{3,2}+\alpha v_{1,1} v_{3,1}+\alpha v_{1,2} v_{3,0}+\mu_{h} v_{1,2}=0, \quad v_{1,3}(0)=0 \\
v_{2,3}^{\prime}+\beta v_{2,2}-\alpha v_{1,2} v_{3,0}-\alpha v_{1,1} v_{3,1}-\alpha v_{1,0} v_{3,2}=0, v_{2,3}(0)=0 \\
v_{3,3}^{\prime}-\gamma v_{2,2}+\gamma v_{2,2} v_{3,0}+\gamma v_{2,1} v_{3,1}+\delta_{1} v_{3,2}+\gamma v_{2,0} v_{3,2}=0, v_{3,3}(0)=0,
\end{gathered}
$$

Solving the differential equations (49)-(54) we get

$$
\begin{aligned}
& v_{1,1}=\int_{0}^{t}\left[-\alpha v_{1,0} v_{3,0}-\mu_{h} v_{1,0}+\mu_{h}\right] d s, \\
& v_{2,1}=\int_{0}^{t}\left[\alpha v_{1,0} v_{3,0}-\beta v_{2,0}\right] d s, \\
& v_{3,1}=\int_{0}^{t}\left[\gamma v_{2,0}-\delta_{1} v_{3,0}-\gamma v_{2,0} v_{3,0}\right] d s, \\
& v_{1,2}=\int_{0}^{t}\left[-\mu_{h} v_{1,1}-\alpha v_{1,1} v_{3,0}-\alpha v_{1,0} v_{3,1}\right] d s, \\
& v_{2,2}=\int_{0}^{t}\left[-\beta v_{2,1}+\alpha v_{1,1} v_{3,0}+\alpha v_{1,0} v_{3,1}\right] d s, \\
& v_{3,2}=\int_{0}^{t}\left[\gamma v_{2,1}-\gamma v_{2,1} v_{3,0}-\delta_{1} v_{3,1}-\gamma v_{2,0} v_{3,1}\right] d s,
\end{aligned}
$$




$$
\begin{aligned}
& v_{1,3}=\int_{0}^{t}\left[\alpha v_{1,0} v_{3,2}-\alpha v_{1,1} v_{3,1}-\alpha v_{1,2} v_{3,0}-\mu_{h} v_{1,2}\right] d s \\
& v_{2,3}=\int_{0}^{t}\left[-\beta v_{2,2}+\alpha v_{1,2} v_{3,0}+\alpha v_{1,1}+\alpha v_{1,0} v_{3,2}\right] d s \\
& v_{3,3}=\int_{0}^{t}\left[\gamma v_{2,2}-\gamma v_{2,2} v_{3,0}-\gamma v_{2,1} v_{3,1}-\delta_{1} v_{3,2}-\gamma v_{2,0} v_{3,2}\right] d s
\end{aligned}
$$

Taking the actual physiological data from the Health Ministry of Indonesia [8], $c_{1}=\frac{7675406}{7675893}, c_{2}=\frac{487}{7675893}, c_{3}=0.056, \quad$ as $\quad$ well as $\alpha=0.232198, \beta=$ $0.328879, \gamma=0.375$, and $\delta_{1}=0.0323$, yields

$$
\begin{aligned}
& v_{1,1}=0.999937-0.0130023 t, \\
& v_{2,1}=0.0000634454+0.0129814 t, \\
& v_{3,1}=0.56-0.00178634 t \\
& v_{1,2}=0.999937-0.0130023 t+0.000292213 t^{2}, \\
& v_{2,2}=0.0000634454+0.0129814 t-0.00242657 t^{2}, \\
& v_{3,3}=0.56-0.00178634 t+0.00232658 t^{2},
\end{aligned}
$$

The 3-term HPM solutions,

$$
\begin{aligned}
& x(t)=\sum_{j=0}^{2} v_{1, j}=0.999937-0.0130023 \mathrm{t}+0.000292213 \mathrm{t}^{2} \\
& y(t)=\sum_{j=0}^{2} v_{2, j}=0.0000634454+0.0129814 \mathrm{t}-0.00242657 \mathrm{t}^{2}, \\
& z(t)=\sum_{j=0}^{2} v_{3, j}=0.056-0.00178634 \mathrm{t}+0.00232658 \mathrm{t}^{2} .
\end{aligned}
$$

In this paper, we calculated the HPM until the tenth term in order to obtain a reliable solution. It can be calculated for more terms to reach the exact solution.

\section{Implementation of VIM}

First, we consider the SIR model which was written in (1)-(3). To apply the VIM to the SIR model, we construct the correction functional as follows:

$$
\begin{aligned}
& x_{n+1}(t)=x_{n}+\int_{0}^{t} \lambda_{1}(s)\left[\frac{d x_{n}}{d s}-\mu_{h}\left(1-x_{n}\right)+\alpha \widetilde{x_{n} z_{n}}\right] d s, \\
& y_{n+1}(t)=y_{n}+\int_{0}^{t} \lambda_{2}(s)\left[\frac{d y_{n}}{d s}-\alpha \widetilde{x_{n} z_{n}}+\beta y_{n}\right] d s, \\
& z_{n+1}(t)=z_{n}+\int_{0}^{t} \lambda_{3}(s)\left[\frac{d z_{n}}{d s}-\gamma y_{n}+\gamma \widetilde{y_{n} z_{n}}+\delta_{1} z_{n}\right] d s,
\end{aligned}
$$


where $\lambda_{i}, \quad i=1,2,3$ are a general Lagrange multiplier which can be identified optimally via the variational theory and the subscript $n$ indicates the $n$ th. To obtain the optimal $\lambda(s)$, we proceed as follows:

$$
\begin{aligned}
& \delta x_{n+1}=\delta x_{n}+\int_{0}^{t} \delta \lambda_{1}(s)\left[\frac{d x_{n}}{d s}-\mu_{h}\left(1-x_{n}\right)+\alpha \widetilde{x_{n} z_{n}}\right] d s, \\
& \delta y_{n+1}=y_{n}+\int_{0}^{t} \delta \lambda_{2}(s)\left[\frac{d y_{n}}{d s}-\alpha \widetilde{x_{n} z_{n}}+\beta \widetilde{y_{n}}\right] d s, \\
& z_{n+1}=z_{n}+\int_{0}^{t} \lambda_{3}(s)\left[\frac{d z_{n}}{d s}-\gamma \widetilde{y_{n}}+\gamma \widetilde{y_{n} z_{n}}+\widetilde{\delta_{1} z_{n}}\right] d s,
\end{aligned}
$$

where $\tilde{x}_{n}, \tilde{y}_{n}$ and $\tilde{z}_{n}$ are considered restricted variations, i.e., $\tilde{x}_{n}, \tilde{y}_{n}=0$ and $\tilde{z}_{n}=0$.

Then, we have

$$
\begin{aligned}
& \delta x_{n+1}=\delta x_{n}+\int_{0}^{t} \delta \lambda_{1}(s)\left[\frac{d x_{n}}{d s}+\mu_{h} x_{n}\right] d s, \\
& \delta y_{n+1}=y_{n}+\int_{0}^{t} \delta \lambda_{2}(s)\left[\frac{d y_{n}}{d s}+\beta y_{n}\right] d s, \\
& \delta z_{n+1}=\delta z_{n}+\int_{0}^{t} \delta \lambda_{3}(s)\left[\frac{d z_{n}}{d s}+\delta_{1} z_{n}\right] d s,
\end{aligned}
$$

or

$$
\begin{aligned}
& \delta x_{n+1}=\delta x_{n}+\int_{0}^{t}\left[\delta \lambda_{1} \frac{d x_{n}}{d s}+\delta \lambda_{1} \mu_{h} x_{n}\right] d s \\
& \delta y_{n+1}=\delta y_{n}+\int_{0}^{t}\left[\delta \lambda_{2}(s) \frac{d y_{n}}{d s}+\delta \lambda_{2} \beta y_{n}\right] d s \\
& \delta z_{n+1}=\delta z_{n}+\int_{0}^{t}\left[\delta \lambda_{3} \frac{d z_{n}}{d s}+\delta \delta_{1} \lambda_{3} z_{n}\right] d s
\end{aligned}
$$

Thus, we obtain the following stationary conditions

$$
\begin{aligned}
& \delta x_{n+1}=\delta\left(1+\lambda_{1}\right) x_{n}+\int_{0}^{t} \delta\left[\lambda_{1}^{\prime}+\lambda_{1} \mu_{h}\right] x_{n} d s \\
& \delta y_{n+1}=\delta\left(1+\lambda_{2}\right) y_{n}+\int_{0}^{t} \delta\left[\lambda_{2}^{\prime}+\lambda_{2} \beta\right] y_{n} d s \\
& \delta z_{n+1}=\delta\left(1+\lambda_{3}\right) z_{n}+\int_{0}^{t} \delta\left[\lambda_{3}^{\prime}+\delta \delta_{1} \lambda_{3}\right] z_{n} d s
\end{aligned}
$$

Thus, we obtain the following stationary conditions

$$
\begin{aligned}
& \delta x_{n}:\left.\left(1-\lambda_{1}(t)\right)\right|_{s=t}=0, \\
& \delta y_{n}:\left.\left(1-\lambda_{2}(t)\right)\right|_{s=t}=0, \\
& \delta z_{n}:\left.\left(1-\lambda_{3}(t)\right)\right|_{s=t}=0, \\
& \delta x_{n}^{\prime}: \lambda_{1}{ }^{\prime}(s)+\left.\mu_{h} \lambda_{1}(s)\right|_{s=t}=0,
\end{aligned}
$$




$$
\begin{array}{ll}
\delta y_{n}^{\prime}: & \lambda_{2}(s)+\left.\beta \lambda_{2}(s)\right|_{s=t}=0, \\
\delta z_{n}^{\prime}: & \lambda_{3}(s)+\left.\delta_{1} \lambda_{3}(s)\right|_{s=t}=0,
\end{array}
$$

Solving this system of equations yields

$$
\begin{aligned}
& \lambda_{1}(s)=-e^{\mu_{h}(s-t)}, \\
& \lambda_{2}(s)=-e^{\beta(s-t)}, \\
& \lambda_{3}(s)=-e^{\delta_{1}(s-t)},
\end{aligned}
$$

Here, the general Lagrange multiplier in (88) is expanded by Taylor series only one term, so the general Lagrange multiplier can be written as follows

$$
\begin{aligned}
& \lambda_{1}(s)=-1, \\
& \lambda_{2}(s)=-1, \\
& \lambda_{3}(s)=-1,
\end{aligned}
$$

Substituting the general Lagrange multiplier in (89) into the correction functional in (73)-(75) results in the following iteration formula:

$$
\begin{aligned}
& x_{n+1}(t)=x_{n}-\int_{0}^{t}\left[\frac{d x_{n}}{d s}-\mu_{h}\left(1-x_{n}\right)+\alpha x_{n} z_{n}\right] d s, \\
& y_{n+1}(t)=y_{n}-\int_{0}^{t}\left[\frac{d y_{n}}{d s}-\alpha x_{n} z_{n}+\beta y_{n}\right] d s, \\
& z_{n+1}(t)=z_{n}-\int_{0}^{t}\left[\frac{d z_{n}}{d s}-\gamma y_{n}+\gamma y_{n} z_{n}+\delta_{1} z_{n}\right] d s .
\end{aligned}
$$

The iteration starts with an initial approximation as provided by the Health ministry of Indonesia [1], $c_{1}=\frac{7675406}{7675893}, c_{2}=\frac{487}{7675893}, c_{3}=0.056$, as well as $\alpha=0.232198, \beta=0.328879, \gamma=0.375$, and $\delta_{1}=0.0323$. The iteration formula (90)-(92) now yields

$$
\begin{aligned}
x_{1}= & 0.9999365546-0.0130022687 t \\
y_{1}= & 0.00006344538675+0.01298140513 t \\
z_{1}= & 0.056-0.001786340333 t \\
x_{2}= & 0.9999365546-0.0130022687 t \\
& +2.922132174 \times 10^{-4} t^{2}-1.797714851 \times 10^{-6} t^{3}, \\
y_{2}= & 0.00006344538675+0.01298140513 t \\
& +0.1797714851 \times 10^{-5} t^{3}-0.002426569924 t^{2}, \\
z_{2}= & 0.056-0.001786340333 t+0.002326579355 t^{2} \\
& +0.2898650945 \times 10^{-5} t^{3}
\end{aligned}
$$




$$
\begin{aligned}
x_{3}= & 0.9999365546-0.01300226807 t \\
& -0.0001831331308 t^{3}+0.0002922132174 t^{2} \\
& +0.1728532016 \times 10^{-12} t^{7} \\
& +0.1290829001 \times 10^{-9} t^{6} \\
& -0.2997118573 \times 10^{-7} t^{5} \\
& +0.1623956764 \times 10^{-5} t^{4} \\
y_{3}= & 0.00006344538675+0.01298140513 t \\
& +0.000449144614 t^{3}-0.00242656993 t^{2} \\
& -0.1728532016 \times 10^{-12} t^{7} \\
& -0.1290829001 \times 10^{-9} t^{6} \\
& +0.2997118573 \times 10^{-7} t^{5} \\
& -0.1771743755 \times 10^{-5} t^{4}, \\
z_{3}= & 0.056-0.00178634033 t-0.000308504557 t^{3} \\
+ & 0.002326579355 t^{2}-0.2791579206 \times 10^{-12} t^{7} \\
+ & 0.1782033109 \times 10^{-9} t^{6} \\
+ & 0.4208392710 \times 10^{-6} t^{3} \\
- & 0.3102165044 \times 10^{-5} t^{4}(101),
\end{aligned}
$$

and so on.

\section{$7 \quad$ Result and Discussion}

The susceptible infected and recovery model was solved. From the data in [1], some parameters were taken $\left(\gamma_{h}\right)=0.3288330,\left(b \beta_{v}\right)=0.3750000,\left(b \beta_{h}\right)=$ $0.7500000,\left(\mu_{h}\right)=0.0000460$, and $\left(\mu_{v}\right)=0.0323000$. The first iteration and term was started by $x(0)=\frac{7675406}{7675893}, y(0)=\frac{487}{7675893}$ and $z(0)=0.056$. The iterative system of the SIR model was coded in a Maple package by restricting the number of significant digits in its environment to 16 . We then displayed the comparison between the RK4 solution and the collected data in [1], see Figure 1. From Figure 1, the RK4 solution with $\Delta t=0.001$ is exactly same as the plotted data shown in [1]. Thus the RK4 solutions can be considered a benchmark for this problem. Figure 2 presents the VIM, HPM and RK4 solutions with $\Delta t=0.001$ for $t \in[0,13]$. From Figure 2 VIM is accurate only for small time intervals, but when the interval was extended, the VIM solution 
diverges for $t \leq 15$. Moreover, when the iteration of the VIM increased, we needed more computer memory to calculate. This means that the method is inefficient. Whereas the HPM is less accurate than the VIM for small time intervals, when the term of the HPM is increased, the HPM solution converges to the RK4 solution and the plot of the collected data [1] at certain times. Figure 3 showed the approximate solution of susceptible populations $(x(t))$, infected population $(y(t))$, and vector population $(z(t))$ via the $10^{\text {th }}$ term HPM and the $10^{\text {th }}$ VIM and RK4 with $\Delta t=0.001$. From the Figures 1,2 , and 3 the HPM solutions are reliable compared to the VIM solution. The VIM solution started to diverge for time interval $[0,15]$. Both the HPM and the RK4 solutions showed good synchronization at the time performed and both results agree very well with each other.

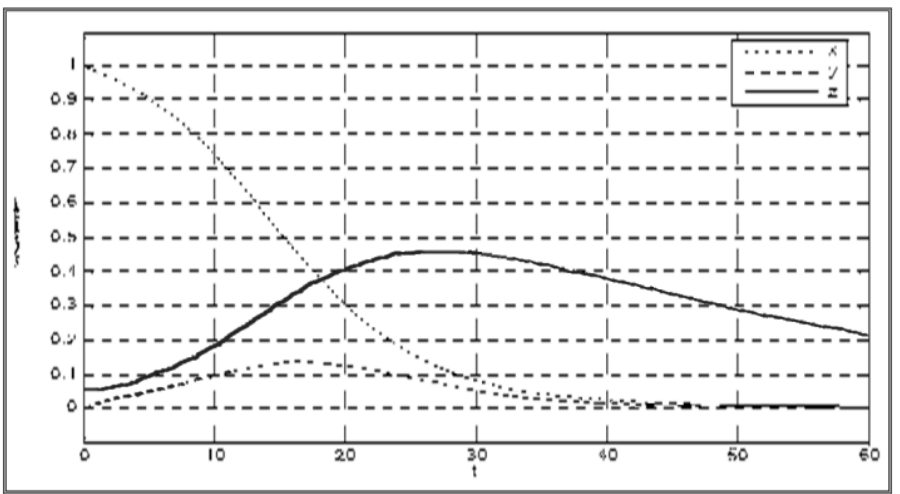

(a)

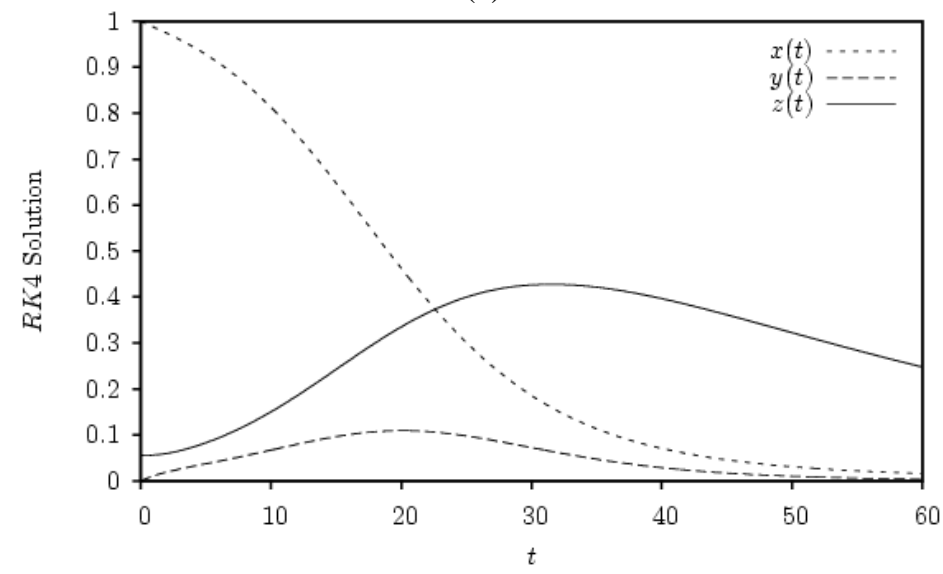

(b)

Figure 1 The succeptible $(\mathrm{x}(\mathrm{t}))$, infected $(\mathrm{y}(\mathrm{t}))$ and removed/recovery $(\mathrm{z}(\mathrm{t}))$ populations using (a) RK4 for $t=0.001$ and (b) ODESOLVE [1]. 


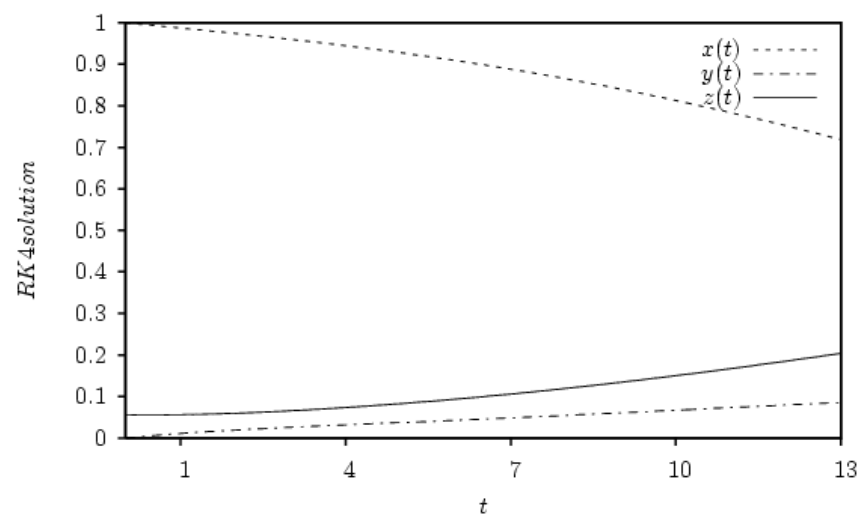

(a)

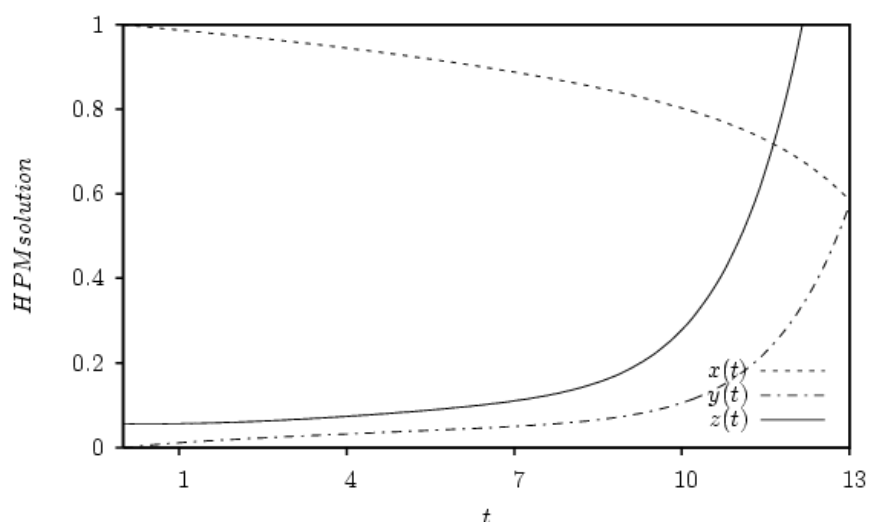

(b)

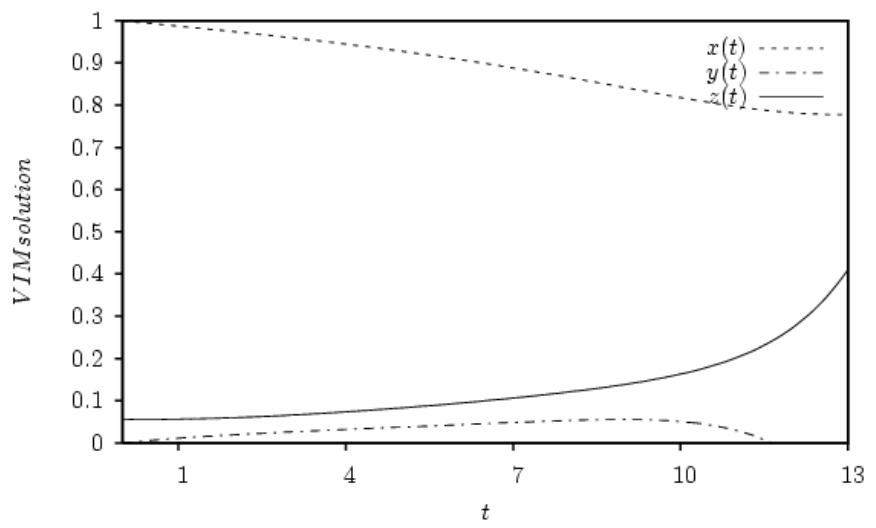

(c)

Figure 2 Approximate solution of susceptible population $(x(t))$, infected population $(y(t))$ and Vector population $(z(t))$ using: (a) RK4 for $t=0.001$; (b) $10^{\text {th }}$ term HPM, and (c) 10th iterate VIM. 


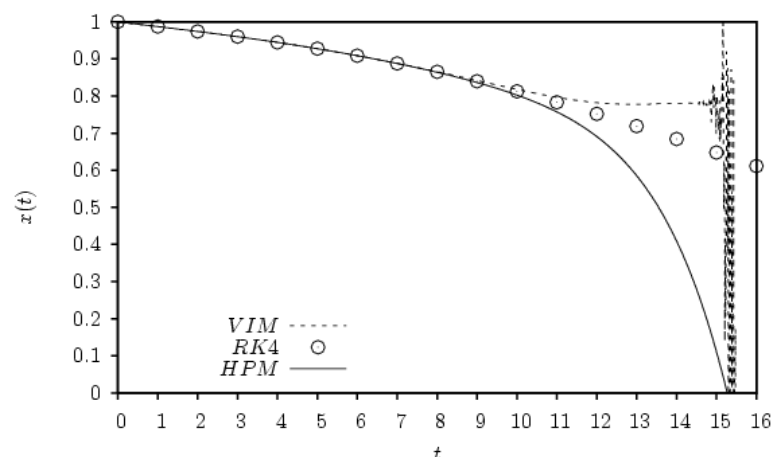

(a)

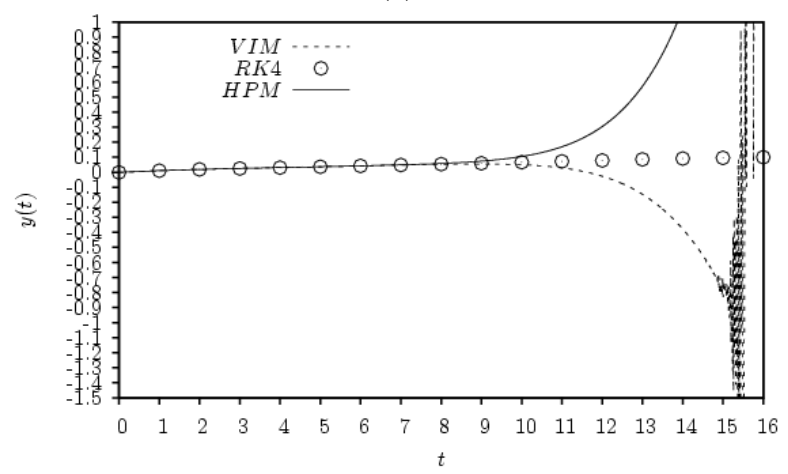

(b)

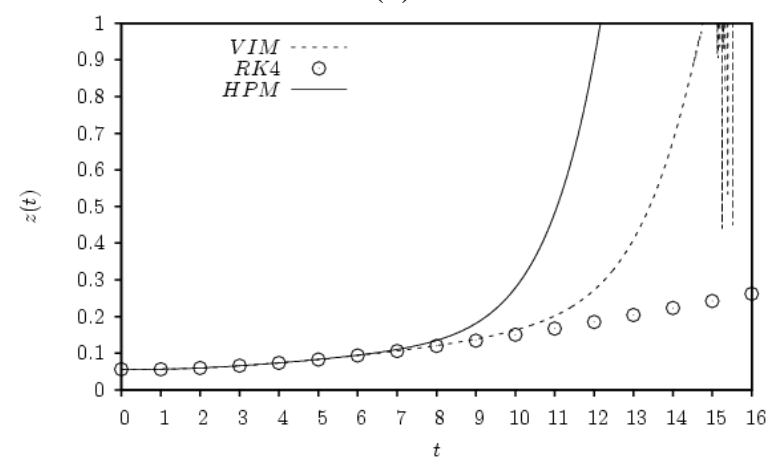

(c)

Figure 3 Approximate solution of: (a) susceptible population $(x(t))$, (b) infected population $(y(t))$ and $(\mathrm{c})$ vector population $(z(t))$ using $10^{\text {th }}$ term HPM, $10^{\text {th }}$ iterate VIM and RK4 for $t=0.001$.

\section{Conclusions}

The approximate of susceptible exposed infected recovery (SEIR) model of dengue fever was investigated. For computations and plots, the Maple and 
Mathematica packages were used. Comparison between homotopy perturbation method (HPM), variational iteration method (VIM) and the fourth-order RungeKutta (RK4) method were made. This work shows that the HPM has much impact on the accuracy and efficacy of the solution in this basic spread of dengue fever. The results anticipated were compared with VIM and the RK4. We found that VIM is accurate only for small time intervals, but when the interval was extended, the VIM solution diverges for $t \leq 15$. Moreover, when the iteration of the VIM increased, we needed more computer memory to calculate. This means that the method is inefficient. Whereas the HPM is less accurate than the VIM for small time intervals, when the term of the HPM is increased, the HPM solution converges to the RK4 solution and the plot of the collected data [1] at certain times. Finally, we conclude that HPM is a very reliable method in solving a broad array of dynamical problems due to its consistency used in a longer time frame.

\section{Acknowledgements}

We gratefully acknowledge the financial support received from Direktorat Jenderal Pendidikan Tinggi (DIKTI) Grant No. 0019/ES.2/PL/2012 (Desentralisasi DP2M,No.020A/UN33.8/KEP/KU 2013).

\section{References}

[1] Side, S. \& Noorani, M.S.M, A SIR Model for Spread of Dengue Fever Disease (Simulation for South Sulawesi, Indonesia and Selangor, Malaysia), World Journal of Modelling and Simulation, 9(2), pp. 96-105, 2013.

[2] Derouich, M. \& Boutayeb, A., Dengue Fever: Mathematical Modelling and Computer Simulation, Appl. Math. Comput., 177(2), pp. 528-544, 2006.

[3] Esteva, L. \& Vargas, C., Analysis of Dengue Disease Transmission Model, Math Biosciences, 150, pp. 131-135, 1998.

[4] Nuraini, N., Soewono, E. \& Sidarto, K., Mathematical Model of Dengue Disease Transmission with Severe dhf Compartment, Bulletin of the Malaysian Mathematical Sciences Society, 30(2), pp.143-157, 2007.

[5] Pongsumpun, P., Transmission Model for Dengue Disease with and without the Effect of Extrinsic Incubation Period, KMITL Sci. Tech., 6, pp. 74-82, 2006.

[6] Soewono, E. \& Supriatna, A.K., A Two-Dimensional Model for the Transmission of Dengue Fever Disease, Bull. Malaysian Matt. Sci. Soc., 24, pp. 49-57, 2005.

[7] Yaacob, Y., Analysis of a Dengue Disease Transmission Model without Immunity, Matematika, 23(2), pp. 75-81, 2007. 
[8] KKRI, Dengue Fever is Still High in South Sulawesi, Tribun Timur Makassar, 2009, http://www.tribunnews.com/ (10 March 2010).

[9] He, J.H., A Coupling Method of a Homotopy Technique and a Perturbation Technique For Non-Linear Problems, International Journal of Non-Linear Mechanics, 35, pp. 37-43, 2000.

[10] He, J., An Elementary Introduction to the Homotopy Perturbation Method, Comput. Math. Appl., 57(3), pp. 410-412, 2009

[11] He, J.H., Variational Iteration Method for Delay Differential Equations, Commun. Nonlinear Sci. Numer. Simulat., 2, pp. 235-236, 1997.

[12] Yulita Molliq, R., Noorani, M.S.M. \& Hashim, I., Variational Iteration Method for Fractional Heat and Wave-Like Equations, Nonlinier Anal. Real World Appl., 10, pp. 1854-1869, 2013.

[13] Yulita Molliq, R., Noorani, M.S.M., Hashim, I. \& Ahmad, R.R., Approximate Solutions of Fractional Zakharov-Kuznetsov Equations by VIM, J. Comput. Appl. Math, 233(2), pp.103-108,2009.

[14] Yulita Molliq, R. \& Noorani, M.S.M., Solving the Fractional RosenauHyman Equation via Variational Iteration Method and Homotopy Perturbation Method, Inter. J. Diff. Eqn., 2012, ID 472030, 2012.

[15] Yulita Molliq, R., Noorani, M.S.M, Ahmad, R.R. \& Alomari, A.K., Modified Step Variational Iteration Method for Solving Fractional Biochemical Reaction Model, Inter. J. Diff. Eqn., 2011, ID 514384, 2011.

[16] Rafei, M., Daniali, H. \& Ganji, D.D., Variational Iteration Method for Solving the Epidemic Model and the Prey And Predator Problem, Appl. Math. Comput., 186(2), pp. 1701-1709, 2007.

[17] Khan, M.A., Islam, S., Ullah, M., Khan, S.A, Zaman, G., Arif, M. \& Sadiq, F.S., Application of Homotopy Perturbation Method to Vector Host Epidemic Model with Non-Linear Incidences, Research J. Recent Sci., 2(6), pp. 90-95, 2013.

[18] Gothi, A.R., Application of Homotopy Perturbation and Variational Iteration Methods to SIR Epidemic Model, J. Mech. Med. Biol., 11, pp. 149-161, 2011.

[19] Islam, S., Saddiq, S.F., Gulzaman, Khan, M.A., Khan, S.A., Ahmad, F. \& Ullah, M., Analytical Solution of an SEIV Epidemic Model by Homotopy Perturbation Method, VFAST Trans. Math., 1(2), pp. 01-07, 2013.

[20] Inokuti, M., Sekine, H. \& Mura, T., General Use of the Lagrange Multiplier in Nonlinear Mathematical Physics, In: Nemat-Nasser, S., ed., Variational Method in the Mechanics of Solids, Pergamon Press, Oxford, pp. 156-162, 1978. 